\title{
Cubic arc-transitive Cayley graphs on Frobenius groups
}

\author{
Hailin Liu \\ School of Mathematics and Statistics, Yunnan University \\ Kunming 650091, P. R. China \\ hailinliuqp@163.com \\ Lei Wang* \\ Beijing International Center for Mathematical Research \\ Peking University, Beijing 100871, P. R. China \\ wanglei-2468@163.com
}

Received 24 March 2017

Accepted 13 June 2017

Published 10 August 2017

Communicated by T. H. Ha

\begin{abstract}
A Cayley graph $\Gamma$ is called arc-transitive if its automorphism group Aut $\Gamma$ is transitive on the set of arcs in $\Gamma$. In this paper, we give a characterization of cubic arc-transitive Cayley graphs on a class of Frobenius groups.
\end{abstract}

Keywords: Arc-transitive graph; Frobenius group; Cayley graph.

Mathematics Subject Classification: 20B25, 05C25

\section{Introduction}

Throughout the paper, graphs considered are simple, connected and undirected. For a graph $\Gamma$, denoted by $V \Gamma, E \Gamma, A \Gamma, \operatorname{val}(\Gamma)$ and Aut $\Gamma$ the vertex set, edge set, arc set, valency and full automorphism group of $\Gamma$, respectively. An $s$-arc of $\Gamma$ is a sequence of vertices $\left(u_{0}, u_{1}, \ldots, u_{s}\right)$ such that $u_{i}$ is adjacent to $u_{i+1}$ and $u_{i-1} \neq u_{i+1}$ for all possible $i$. For a subgroup $G \leq \operatorname{Aut} \Gamma, \Gamma$ is said to be $(G, s)$-arc-transitive if $G$ is transitive on the set of $s$-arcs in $\Gamma$. In particular, a 0 -arc is a called vertex, a 1 -arc is called an arc for short, and a $(G, 1)$-arc-transitive graph is called $G$-arc transitive graph. As we all know, a graph $\Gamma$ is $G$-arc-transitive if and only if $G$ is transitive on $V \Gamma$ and the vertex stabilizer $G_{v}$ of $v \in V \Gamma$ in $G$ is transitive on the neighborhood $\Gamma(v)$ of $v$.

A graph $\Gamma$ is called a Cayley graph if there exists a group $G$ and a subset $S \subset G \backslash\{1\}$ with $S=S^{-1}:=\left\{g^{-1} \mid g \in S\right\}$ such that the vertices of $\Gamma$ may be

\footnotetext{
* Corresponding author.
} 
identified with the elements of $G$ in such a way that $x$ is adjacent to $y$ if and only if $y x^{-1} \in S$. The Cayley graph $\Gamma$ is denoted by Cay $(G, S)$. Throughout this paper, denote by 1 the vertex of Cay $(G, S)$ corresponding to the identity of $G$.

A graph $\Gamma$ is a Cayley graph on $G$ if and only if Aut $\Gamma$ contains a subgroup which is regular on vertices and isomorphic to $G$. It is well-known that a Cayley graph Cay $(G, S)$ is vertex-transitive. However, a Cayley graph is of course not necessarily arc-transitive. Thus much excellent work has dealt with arc-transitive Cayley graphs. In particular, there are many results about cubic Cayley graphs. For instance, see [11, 16, 17] for cubic symmetric Cayley graphs on finite nonabelian simple groups, which are normal except for $A_{47}$, see [12 for a characterization of connected cubic $s$-transitive Cayley graphs, and see [5] for a classification of the connected arc-transitive cubic Cayley graphs on $\operatorname{PSL}(2, p)$ where $p \geq 5$ is a prime. The objective of this paper is to give a characterization of cubic arc-transitive Cayley graphs on a class of Frobenius groups.

A group $G$ is said to be a Frobenius group if $G$ has the form $G=W: H$ such that $x y \neq y x$ for any $x \in W \backslash\{1\}$ and $y \in H \backslash\{1\}$. In particular, $G$ is called a primitive Frobenius group if $H$ acts irreducibly on $W$.

Theorem 1.1. Let $G=W: H \cong \mathbb{Z}_{p}^{d}: \mathbb{Z}_{n}$ be a primitive Frobenius group, where $p$ is a prime, and $d, n$ are positive integers. Assume that $\Gamma$ is a connected cubic $X$ arc-transitive Cayley graph on $G$, where $G \leq X \leq$ Aut $\Gamma$. Then one of the following statements holds:

(i) $G$ is normal in $X$, and $X_{1}=\mathbb{Z}_{3}$ or $\mathrm{S}_{3}$;

(ii) $G \cong \mathrm{D}_{6}, \Gamma \cong K_{3,3}$, and Aut $\Gamma \cong \mathrm{S}_{3}<\mathrm{S}_{2}$;

(iii) $\Gamma_{W}=\mathrm{K}_{\mathbf{3}, \mathbf{3}}, G \cong \mathbb{Z}_{p}: \mathbb{Z}_{6}$ and $X=(W \times L): H$, where $L \cong \mathbb{Z}_{3}, \mathbf{Z}(X)=1$, and $\Gamma$ is constructed as in Construction 3.1 .

(iv) $\Gamma_{W}=\mathrm{K}_{\mathbf{3}, \mathbf{3}}, G \cong \mathbb{Z}_{p}^{2}: \mathbb{Z}_{6}$ and $X=((W \times L): H): M$, where $p \equiv-1(\bmod 3)$, $L \cong \mathbb{Z}_{3}, M \cong \mathbb{Z}_{2}$ or $1, \mathbf{Z}(X)=1$, and $\Gamma$ is constructed as in Construction 3.3 ,

(v) $\Gamma$ is isomorphic to one of the graphs listed in Table 1 and $\left(G, \operatorname{Aut} \Gamma,(\text { Aut } \Gamma)_{1}\right)$ is as follows.

Remarks on Theorem [1.1, (a) The Cayley graph $\Gamma$ in part (i) is called a $X$ normal Cayley graph. Furthermore, if $X=A u t \Gamma$, then $\Gamma$ is called a normal Cayley graph, introduced in [15.

(b) $H$ acts irreducibly on $W$ if and only if $n$ does not divide $p^{m}-1$ where $m<d$. We call such $n$ a primitive divisor of $p^{d}-1$, refer to [4] Proposition 2.3].

Table 1. Some exceptions.

\begin{tabular}{l|ccc}
\hline$\Gamma$ & Example 3.5 & Example 3.6 & Example 3.5 \\
\hline$G$ & $\mathrm{D}_{14}$ & $\mathbb{Z}_{11}: \mathbb{Z}_{10}$ & $\mathbb{Z}_{23}: \mathbb{Z}_{22}$ \\
\hline Aut $\Gamma$ & $\mathrm{PGL}(2,7)$ & $\mathrm{PGL}(2,11)$ & PGL $(2,23)$ \\
\hline (Aut $\Gamma)_{1}$ & $\mathrm{~S}_{4}$ & $\mathrm{D}_{12}$ & $\mathrm{~S}_{4}$ \\
\hline
\end{tabular}


After this introductory section, some preliminary results are given in Sec. 2 and a few examples appearing in Theorem 1.1 are given in Sec. 3. Then we complete the proof of Theorem 1.1 in Sec. 4

\section{Preliminary Results}

In this section, we quote some preliminary results, which will be used in the subsequent sections.

Let $\Gamma=(V, E)$ be a $G$-vertex-transitive graph. Then, for $\alpha \in V$, the stabilizer $G_{\alpha}$ is a core-free subgroup in $G$, that is, $\bigcap_{g \in G} G_{\alpha}^{g}=1$. Set $H=G_{\alpha}$ and $D=\left\{x \mid \alpha^{x} \in\right.$ $\Gamma(\alpha)\}$, where $\Gamma(\alpha)$ is the set of neighbors of $\alpha$ in $\Gamma$. Then $D$ is a union of several double cosets $H x H$. Moreover, $\Gamma$ is isomorphic to the coset graph $\operatorname{Cos}(G, H, D)$ defined over $\{H x \mid x \in G\}$ with edge set $\left\{\left\{H g_{1}, H g_{2}\right\} \mid g_{2} g_{1}^{-1} \in D\right\}$.

The following statements for coset graphs are well known.

(a) $\Gamma$ is undirected if and only if $D=D^{-1}:=\left\{x^{-1} \mid x \in D\right\}$.

(b) $\Gamma$ is connected if and only if $\langle H, D\rangle=G$.

(c) $\Gamma$ is $G$-arc-transitive if and only if $D=H g H$ for $g \in G$ with $g^{2} \in H$; moreover, $g$ can be chosen as a 2-element with $g \in \mathrm{N}_{G}\left(H \cap H^{g}\right)$ and $g^{2} \in H \cap H^{g}$.

For a Cayley graph $\Gamma=\operatorname{Cay}(G, S)$. Let $\operatorname{Aut}(G, S)=\left\{\alpha \in \operatorname{Aut}(G) \mid S^{\alpha}=S\right\}$. Then we have the following basic result.

Lemma 2.1 ([7, Lemma 2.1]). Let $\Gamma=\operatorname{Cay}(G, S)$ be a Cayley graph. Then the normalizer $\mathrm{N}_{\text {Aut } \Gamma}(G)=G$ :Aut $(G, S)$.

The vertex stabilizer for $s$-arc-transitive graphs of valency 3 is known.

Lemma 2.2 (Tutte). Let $\Gamma$ be a connected $(G, s)$-transitive cubic graph, where $s \geq 1$. Then $s \leq 5$ and the stabilizer $G_{u}$ and $\left|G_{u}\right|$ satisfy the following table:

\begin{tabular}{c|ccccc}
\hline$s$ & 1 & 2 & 3 & 4 & 5 \\
\hline$G_{u}$ & $\mathbb{Z}_{3}$ & $\mathrm{~S}_{3}$ & $\mathrm{D}_{12}$ & $\mathrm{~S}_{4}$ & $\mathrm{~S}_{4} \times \mathrm{S}_{2}$ \\
\hline$\left|G_{u}\right|$ & 3 & $2 \cdot 3$ & $2^{2} \cdot 3$ & $2^{3} \cdot 3$ & $2^{4} \cdot 3$ \\
\hline
\end{tabular}

We end this section with two basic results for cubic arc-transitive graph.

Lemma 2.3. Let $G$ be a finite group. Let $\Gamma=\operatorname{Cay}(G, S)$ be connected of valency 3. Assume that Aut $\Gamma$ has a subgroup $X$ such that $\Gamma$ is $X$-arc-transitive and $G \unlhd X$. Then $X_{1}=\mathbb{Z}_{3}$ or $\mathrm{S}_{3}$.

Proof. Since $\Gamma$ is connected, we have $G=\langle S\rangle$, and thus $\operatorname{Aut}(G, S)$ acts faithfully on $S$. So $\operatorname{Aut}(G, S) \lesssim \mathrm{S}_{3}$. By Lemma 2.1 we have $X \leq \mathrm{N}_{\text {Aut } \Gamma}(G)=G$ :Aut $(G, S)$. Thus $X_{1} \leq \operatorname{Aut}(G, S) \lesssim \mathrm{S}_{3}$. Since $X_{1}$ is transitive on $S$, it follows that $X_{1} \cong \mathbb{Z}_{3}$ or $\mathrm{S}_{3}$. 
Lemma 2.4. Let $\Gamma$ is a connected cubic $X$-arc-transitive graph, and let $N \triangleleft X$ such that $X / N$ is insoluble. Then $\Gamma$ is a normal cover of $\Gamma_{N}$.

Proof. Let $u \in V \Gamma$. Let $B=u^{N}$ be an orbit of $N$ acting on $V \Gamma$. By Lemma [2.2, $X_{u}$ is a $\{2,3\}$-group, and hence $X_{u}$ is soluble. Let $K$ be the kernel of $X$ acting on $V \Gamma_{N}$. Then $K_{u}$ is soluble as $K_{u} \unlhd X_{u}$. By the Frattini argument, we have that $K=N K_{u}$. Note that $K / N \cong N K_{u} / N \cong K_{u} /\left(N \cap K_{u}\right)$, it follows that $K / N$ is soluble. Since $X / N$ is insoluble, $X / K \cong(X / N) /(K / N)$ is insoluble. Thus $\Gamma$ is a normal cover of $\Gamma_{N}$.

\section{Examples}

In this section, we give some examples of graphs satisfying Theorem 1.1.

Construction 3.1. Let $G=W: H=\mathbb{Z}_{p}: \mathbb{Z}_{6}$ be a Frobenius group, where $p$ is a prime. Let $X=(W \times L): H$, where $L \cong \mathbb{Z}_{3}$ and $\mathbf{Z}(X)=1$. Write $H=\langle h\rangle$ and $L=\langle a\rangle$.

Let $N=\left\langle a h^{2}\right\rangle$ and $g=w a h^{3}$, where $1 \neq w \in W$. Set

$$
\Gamma=\operatorname{Cos}(X, N, N g N) \text {. }
$$

The next lemma shows that the graphs constructed here are as required.

Lemma 3.2. Let $\Gamma$ be a graph constructed in Construction 3.1. Then $\Gamma$ is a connected cubic $X$-arc-transitive Cayley graph on $G$, and $G$ is not normal in $X$. In particular, $\Gamma$ is a cover of $\Gamma_{W}$, and $\Gamma_{W} \cong \mathrm{K}_{\mathbf{3}, \mathbf{3}}$.

Proof. By the definition, $N$ is core-free in $X$, and hence $X \leq$ Aut $\Gamma$. Now $X=G N$ and $G \cap N=1$, and thus $G$ acts regularly on the vertex set $[X: N]$. So $\Gamma$ is a Cayley graph of $G$. Obviously, $G$ is not normal in $X$.

Let $Y=\langle N, g\rangle$. Let $n=a h^{2}$. Since $\mathbf{Z}(X)=1$, we have $a^{h}=a^{-1}$. Then $g^{n}=w^{h^{2}} a^{-1} h^{3}$, and $g^{n} g=w^{h^{2}} w^{-1} a^{-2}$. Let $x=w^{h^{2}} w^{-1}$. Since $h$ acts fixed-pointfree on $W$, it follows that $x \neq 1$. Clearly, $\left(g^{n} g\right)^{3}=x^{3} \neq 1$. Thus $x \in Y$, and $W \leq Y$. So $X=Y$. It is easy to show that $N \cap N^{g}=1$. Therefore, $\Gamma$ is a connected cubic $X$-arc-transitive Cayley graph. Since $\Gamma$ is of valency $3, \Gamma$ is a cover of $\Gamma_{W}$. Clearly, $\Gamma_{W}$ is a Cayley graph of $G / W$. By [1, Theorem 1.2], we obtain $\Gamma_{W} \cong \mathrm{K}_{3,3}$.

Construction 3.3. Let $G=W: H=\mathbb{Z}_{p}^{2}: \mathbb{Z}_{6}$ be a Frobenius group, where $p \equiv-1$ $(\bmod 3)$ is a prime. Let $X=(W \times L): H$, where $L \cong \mathbb{Z}_{3}$ and $\mathbf{Z}(X)=1$. Write $H=\langle h\rangle$ and $L=\langle a\rangle$.

Let $N=\left\langle a h^{2}\right\rangle$ and $g=w a h^{3}$, where $1 \neq w \in W$. Set

$$
\Gamma=\operatorname{Cos}(X, N, N g N) \text {. }
$$

Arguing similarly as Lemma 3.2, we have the following lemma.

Lemma 3.4. Let $\Gamma$ be a graph constructed in Construction 3.3. Then $\Gamma$ is a connected cubic $X$-arc-transitive Cayley graph on $G$, and $G$ is not normal in $X$. In particular, $\Gamma$ is a cover of $\Gamma_{W}$, and $\Gamma_{W} \cong \mathrm{K}_{\mathbf{3}, \mathbf{3}}$. 
Applying [13, Examples 3.6 and 3.8], we have the following two examples.

Example 3.5. Let $T=\operatorname{PSL}(2, p)$, and let $X=\operatorname{PGL}(2, p)$, where $p=7$ or 23 . Take a subgroup $H \cong \mathrm{S}_{4}$ of $T$, and let $K \cong \mathrm{D}_{8}$ be a Sylow 2-subgroup of $H$. Then $\mathrm{N}_{G}(K)=\mathrm{D}_{16}$ and let $g \in \mathrm{N}_{G}(K) \backslash K$ be an involution such that $\langle H, g\rangle=X$. Let $G=\mathrm{D}_{14}$ or $\mathbb{Z}_{23}: \mathbb{Z}_{22}$. Then the coset graph $\Gamma=\operatorname{Cos}(X, H, H g H)$ is a Cayely graph on $G$ with full automorphism group $\operatorname{PGL}(2, p)$.

Example 3.6. Let $T=\operatorname{PSL}(2,11)$, and let $X=\operatorname{PGL}(2,11)$. Take a subgroup $H \cong \mathrm{D}_{12}$ of $T$, and let $K \cong \mathbb{Z}_{2}$ be a Sylow 2-subgroup of $H$. Then $\mathrm{N}_{G}(K)=\mathrm{S}_{4}$. Let $g \in \mathrm{N}_{G}(K) \backslash K$ be an involution such that $\langle H, g\rangle=X$. Then the coset graph $\Gamma_{1}=\operatorname{Cos}(X, H, H g H)$ is a Cayely graph on $\mathbb{Z}_{11}: \mathbb{Z}_{10}$ with full automorphism group $\operatorname{PGL}(2,11)$.

\section{Proof of Theorem 1.1}

In this section, we will prove Theorem 1.1 by a series of lemmas.

Let $G=W: H \cong \mathbb{Z}_{p}^{d}: \mathbb{Z}_{n}$ be a primitive Frobenius group, where $p$ is a prime, and $d, n$ are integers. Assume that $\Gamma=\operatorname{Cay}(G, S)$ is a connected cubic $X$-arc-transitive Cayley graph on $G$, where $G \leq X \leq$ Aut $\Gamma$. First of all, we study the case where the automorphism group $X$ is soluble.

For $G=\mathrm{D}_{2 p}$, we have the following lemma, see [13 Theorem 1.1].

Lemma 4.1. Let $G$ be the dihedral group of order $2 p$, and let $\Gamma$ be a connected cubic $X$-arc-transitive Cayley graph on $G$. Set $G=\langle a, b\rangle$ with $o(a)=p, o(b)=2$, and $a^{b}=a^{-1}$. Assume that $X$ is soluble. Then $\Gamma=\operatorname{Cay}(G, S)$ for one of the following subsets $S$ of $G$.

(i) $p=3$ and $S=\left\{a b, a^{2} b, b\right\}$; in this case, $\Gamma \cong \mathrm{K}_{\mathbf{3}, \mathbf{3}}$;

(ii) $p>3$ and $S=\left\{a b, a^{k} b, b\right\}$, where $k^{2}-k+1 \equiv 0(\bmod p)$; in this case, Aut $\Gamma \cong$ $\mathrm{D}_{2 p}: \mathbb{Z}_{3}$.

Lemma 4.2. Let $B=\mathrm{S}_{4}$. Then $B \not \mathrm{GL}(2, p)$, where $p$ is a prime.

Proof. If $p=2$, then the statement follows. In the following, assume that $p$ is odd.

Let $H=\mathrm{GL}(2, p)$, and $L=\mathrm{SL}(2, p)$. Suppose that $B \leq H$. By [8, Theorem 8.3, p. 42], $L$ has a unique involution. Thus $B \cap L=1$. It follows that $B /(B \cap L) \lesssim$ $H / L \lesssim \mathbb{Z}_{p-1}$, which is a contradiction. Thus $B \not \leq H$.

Let $F$ be the Fitting subgroup of $X$, that is, $F$ is the largest nilpotent normal subgroup of $X$. Then $F \neq 1$ and $\mathrm{C}_{X}(F) \leq F$ as $X$ is soluble, and $F=\left\langle\mathbf{O}_{p}(X)|p||X|\right\rangle$.

For a group $H$ and a prime $p$, by $H_{p}$ we mean a Sylow $p$-subgroup of $H$.

Lemma 4.3. If $G \supsetneqq \mathrm{D}_{2 p}$, then $W$ is normal in $X$. 
Proof. We first show that $G \cap F \neq 1$. Suppose that $G \cap F=1$. Since $X=G X_{u}$, we have $|F|\left\|X_{u}\right\| 48$, see Lemma [2.2, where $u \in V \Gamma$.

Assume that $F$ is transitive on $V \Gamma$. Then $|G||| F \mid$. Since $H$ acts irreducibly on $W$, we conclude that $G \cong \mathbb{Z}_{2}^{2}: \mathbb{Z}_{3}$. However, there exists no cubic symmetric graphs of order 12, see [6, Theorem 6.2]. Similarly, we also exclude the case where $\Gamma_{F}=K_{2}$.

Thus $\Gamma$ is a normal cover of $\Gamma_{F}$. Then $|F|$ divides $|G|$. Since $\mathrm{C}_{G}(F) \leq F$, we conclude that $\mathrm{C}_{G}(F)=1$. This implies that $F$ is noncyclic. Thus 2||$F \mid$.

Suppose that $p=2$. By the previous paragraph, we have $n \geq 5$. Assume that $\Phi(F)=1$. Since $|F|$ divides both 48 and $|G|$, we obtain $F_{2} \cong \mathbb{Z}_{2}^{k}$ and $F_{2^{\prime}} \cong \mathbb{Z}_{3}$ or 1 , where $k \leq \operatorname{gcd}(4, d)$. Note that $\mathrm{C}_{X}(F) \leq F$, it follows that $G \leq \mathrm{GL}(k, 2)$. By Atlas 3], there exists no $k$ and $G$ satisfying the above relation. Thus $\Phi(F) \neq 1$. Let $\bar{F}=F / \Phi(F)$ and $\bar{H}=H \Phi(F) / \Phi(F)$. Then $\bar{H} \cong H$. If $\mathrm{C}_{\bar{H}}(\bar{F}) \neq 1$, then $\mathrm{C}_{H}(F) \neq 1$, see [8, Theorem 1.4, p. 174]. Thus $\mathrm{C}_{\bar{H}}(\bar{F})=1$. Clearly, $\left|\bar{F}_{2}\right| \leq 8$. By Atlas $[3], \bar{F}_{2} \cong \mathbb{Z}_{2}^{3}$ and $\bar{H} \cong \mathbb{Z}_{7}$. For this case $d=3$, and $\left|F_{2}\right|=16$, which is a contradiction.

Suppose that $p$ is odd. Assume $\Phi(F)=1$. Then $F_{2} \cong \mathbb{Z}_{2}^{k}$, where $k \leq 4$ and $2^{k} \mid n$. As $\mathrm{C}_{X}(F) \leq F$, we have $G \leq \mathrm{GL}(k, 2)$. It follows that $W \leq \mathrm{GL}(k, 2)$, and $\mathbb{Z}_{2^{k}} \leq$ $\mathrm{GL}(k, 2)$. By Atlas [3], there exists no $k$ and $W$ satisfying the above relation. Thus $\Phi(F) \neq 1$. Since $F_{3} \leq \mathbb{Z}_{3}$, we conclude that $\Phi(F) \leq F_{2}$. Let $\bar{W}=W \Phi(F) / \Phi(F)$ and $\bar{F}_{2}=F_{2} / \Phi(F)$. If $\mathrm{C}_{\bar{W}}\left(\bar{F}_{2}\right) \neq 1$, then $\mathrm{C}_{W}\left(F_{2}\right) \neq 1$, refer to [8, Theorem 1.4 , p. 174]. Thus $\mathrm{C}_{\bar{W}}\left(\bar{F}_{2}\right)=1$. Clearly, $\bar{F}_{2} \cong \mathbb{Z}_{2}^{3}$. By Atlas [3], this is impossible.

To sum up, $F \cap G \neq 1$. Since $W$ is minimal in $G$, we conclude that $W \leq F$. Recall that $G \not \mathrm{D}_{2 p}$, it follows that $\Gamma$ is a cover of $\Gamma_{F_{p}}$, and thus $W=F_{p}$. That is to say, $W$ is normal in $X$. This completes the proof.

Lemma 4.4. With the hypothesis of Lemma 4.3, then one of the following statements holds:

(a) $G$ is normal in $X$, and $X_{u}=\mathbb{Z}_{3}$ or $\mathrm{S}_{3}$, where $u \in V \Gamma$;

(b) $\Gamma_{W}=\mathrm{K}_{\mathbf{3}, 3}, G \cong \mathbb{Z}_{p}: \mathbb{Z}_{6}$, and $X=(W \times L): H$, where $L \cong \mathbb{Z}_{3}$, and $\mathbf{Z}(X)=1$;

(c) $\Gamma_{W}=\mathrm{K}_{\mathbf{3}, 3}, G \cong \mathbb{Z}_{p}^{2}: \mathbb{Z}_{6}$, and $X=((W \times L): H): M$, where $p \equiv-1(\bmod 3)$, $L \cong \mathbb{Z}_{3}, M \cong \mathbb{Z}_{2}$ or 1 , and $\mathbf{Z}(X)=1$.

Proof. By Lemma 4.3, $W$ is normal in $X$. Since $G ¥ \mathrm{D}_{2 p}$, we have $n>2$. Then $\Gamma$ is a normal cover of $\Gamma_{W}$. By [1, Theorem 1.2], either $G / W \unlhd X / W$ or $\Gamma_{W}=\mathrm{K}_{4}$ or $\mathrm{K}_{\mathbf{3}, \mathbf{3}}$. In the former case, $G \unlhd X$. By Lemma 2.3, $X_{u}=\mathbb{Z}_{3}$ or $\mathrm{S}_{3}$, where $u \in V \Gamma$. If $\Gamma_{W}=\mathrm{K}_{4}$, then $G / W \cong \mathbb{Z}_{4}$. Note that $\Gamma$ is $X$-arc-transitive, we conclude that $X \cong$ $\mathbb{Z}_{p}^{2} \cdot \mathrm{S}_{4}$. Since $H \cong \mathbb{Z}_{4}$, we have $X / W \cong \mathrm{S}_{4}$ and $X / W \leq \mathrm{GL}(2, p)$, a contradiction, see Lemma 4.2

Now assume that $\Gamma_{W}=\mathrm{K}_{\mathbf{3}, \mathbf{3}}$. Then $G / W \cong \mathbb{Z}_{6}$. Since $G$ is a Frobenius group, $p$ is coprime to 6 . Note that $\Gamma$ is $X$-arc-transitive, we obtain that 3 divides $\left|X_{u}\right|$ by Lemma 2.2. Thus $X_{3} \cong \mathbb{Z}_{3}^{2}$. By Schur-Zassenhaus's Theorem, $X=W:\left(X_{3}: X_{2}\right)$, where $X_{3}: X_{2} \lesssim \mathrm{S}_{3}$ < $\mathrm{S}_{2}$. If $X_{2} \cong \mathrm{D}_{8}$, then $X_{3}$ is a unique minimal normal subgroup of $X_{3}: X_{2} \cong \mathrm{S}_{3}<\mathrm{S}_{2}$. Since $H \cong \mathbb{Z}_{6}$, we conclude that $\mathrm{C}_{X_{3}}(W)=1$. It follows 
that $W \cong \mathbb{Z}_{p}^{2}$, and $X_{3}: X_{2} \lesssim \mathrm{GL}(2, p)$. Recall that $H$ acts irreducibly on $W$, we have $p \equiv-1(\bmod 3)$. By [9] Theorem 7.3, p. 187], $\mathrm{C}_{\mathrm{GL}(2, p)}\left(H_{3}\right) \cong \mathbb{Z}_{p^{2}-1}$. However $\mathrm{C}_{X}\left(H_{3}\right) \geq X_{3}$, a contradiction. Thus $X_{2}=\mathbb{Z}_{2}^{\ell}$, where $\ell=1$ or 2 . By the above argument, we also obtain that $\mathrm{C}_{X_{3}}(W) \cong \mathbb{Z}_{3}$. Recall that $H \cong \mathbb{Z}_{6}$, and $H$ acts irreducibly on $W$, it follows that $W \cong \mathbb{Z}_{p}$ or $\mathbb{Z}_{p}^{2}$. For the former, $p \equiv 1(\bmod 3)$, and for the latter, $p \equiv-1(\bmod 3)$.

Assume that $G \cong \mathbb{Z}_{p}: \mathbb{Z}_{6}$. Then $X_{2} \cong \mathbb{Z}_{2}$, and so $X=(W \times L): H$, where $L \cong \mathbb{Z}_{3}$. Write $\Gamma=\operatorname{Cos}\left(X, X_{u}, X_{u} g X_{u}\right)$, where $X_{u} \cong \mathbb{Z}_{3}, u \in V \Gamma$ and $o(g)=2$. Let $H=\langle h\rangle$. By Sylow's Theorem, we may assume $Y_{u}=\left\langle l h^{2}\right\rangle$, where $l \in L$. If $h$ centralizes $L$, then $g=w h^{3}$, where $w \in W$. It is easily shown that $\left\langle Y_{u}, g\right\rangle=W:\left\langle l^{2} h\right\rangle\langle X$. So $\Gamma$ is not connected. Thus $\mathbf{Z}(X)=1$.

Assume that $G \cong \mathbb{Z}_{p}^{2}: \mathbb{Z}_{6}$. Then $X_{2} \cong \mathbb{Z}_{2}$ or $\mathbb{Z}_{2}^{2}$. Thus $X=((W \times L): H): M$, where $L \cong \mathbb{Z}_{3}$ and $M \cong \mathbb{Z}_{2}$ or 1 . Arguing as above, $L$ does not centralize $H$. Since $\mathrm{S}_{3}^{2}$ has no abelian subgroup of order 12 , it follows that $M$ does not centralize $H_{3}$. So $\mathbf{Z}(X)=1$.

This completes the proof of Lemma 4.4 .

In the remaining section, we study the case where the automorphism group $X$ is insoluble. Denote by $R$ the radical of $X$, that is, $R$ is the largest soluble normal subgroup of $X$. We will deal with the case where $R=1$.

Lemma 4.5. If $R=1$, then, for $u \in V \Gamma,\left(G\right.$, Aut $\left.\Gamma,(\text { Aut } \Gamma)_{u}\right)$ is one of the triples listed in Table 1

Proof. Let $N$ be a minimal normal subgroup of $X$. Since $R=1$, we have $N=$ $T_{1} \times \cdots \times T_{\ell} \cong T^{\ell}$, where $T_{i} \cong T$ is nonabelian simple. By [10], $T$ is one of the following:

$\operatorname{PSL}(4,2), \operatorname{PSU}(3,8), \mathrm{M}_{11}, \operatorname{PSp}(4,3), \operatorname{PSL}(3, q)(q<9), \operatorname{PSL}(2, q)(q>3)$.

Let $W_{i}=T_{i} \cap W$, where $1 \leq i \leq \ell$. Let $L=N \cap H$. Since $G$ is a Frobenius group, it follows that $L$ is a diagonal subgroup of $N$. Write $L=\left\langle h_{1} \cdots h_{\ell}\right\rangle$, where $\left\langle h_{i}\right\rangle \cong L$. Let $H_{i}=\left\langle h_{i}\right\rangle$ and $G_{i}=W_{i}: H_{i}$, where $1 \leq i \leq \ell$. Then $G_{i}$ is a Frobenius group. Let $m=\frac{\left|T_{1}\right|}{\left|G_{1}\right|} \prod_{i=2}^{\ell} \frac{\left|T_{i}\right|}{\left|W_{i}\right|}$. Since $N \cap G=W: L$, and $\frac{|N|}{|N \cap G|}$ divides $\left|X_{u}\right|$, it follows that $m=\frac{|N|}{|N \cap G|}$, and $m|| X_{u} \mid$.

Suppose that $T=\operatorname{PSL}(4,2)$. By Atlas [3], $G_{i} \lesssim A_{7}$. Clearly, 105 does not divide $\left|G_{i}\right|$. So $m$ does not divide $\left|X_{u}\right|$, which is a contradiction. Arguing similarly as above, we exclude the cases where $T=\operatorname{PSU}(3,8), \mathrm{M}_{11}, \operatorname{PSp}(4,3)$, and $\operatorname{PSL}(3, q)(q<9)$.

Suppose that $T=\operatorname{PSL}(2, q)$ where $q=r^{e}$. According to [14, Theorem 6.25], $G_{i}$ is a subgroup of one of the following groups:

$$
\mathbb{Z}_{r}^{e}: \mathbb{Z}_{\frac{\left(r^{e}-1\right)}{d}}, \mathrm{D}_{\frac{2\left(r_{ \pm} e_{1}\right)}{d}}, \mathrm{~A}_{5} \text { and } \operatorname{PGL}\left(2, r^{f}\right),
$$

where $d=(2, r-1)$, and $f \mid e$. In the following, we process our analysis by several cases. 
Case 1: Suppose that $G_{i} \leq \mathbb{Z}_{r}^{e}: \mathbb{Z}_{\frac{(r e-1)}{d}}$. If $\ell>1$, then $(q+1)^{2}(q-1)$ divides 48 since $m$ divides $\left|X_{u}\right|$. It follows that $(q+1)^{2}$ divides 16 , and thus $q \leq 3$, which is a contradiction. Thus $\ell=1$, namely, $N=T$. For this case, $q+1$ divides 48 . Calculation shows that $r^{e}=q=5,7,11$ or 23 . Since $W=\mathbb{Z}_{r}$, we conclude that $W=\mathbb{Z}_{5}, \mathbb{Z}_{7}, \mathbb{Z}_{11}$ or $\mathbb{Z}_{23}$.

Assume that $W=\mathbb{Z}_{5}$. Then $G=\mathbb{Z}_{5}: \mathbb{Z}_{2}$ or $\mathbb{Z}_{5}: \mathbb{Z}_{4}$. By [2, Table [1], there exists no cubic Cayley graphs of order 10, the former case does not occur. For the latter case $\operatorname{Aut}(\operatorname{Cay}(G, S))$ is soluble, this case is also excluded, see [18, Lemma 2.2].

Assume that $W=\mathbb{Z}_{7}, \mathbb{Z}_{11}$ or $\mathbb{Z}_{23}$. Then $G$ is one of the following

$$
\mathrm{D}_{14}, \quad \mathbb{Z}_{7}: \mathbb{Z}_{6}, \quad \mathrm{D}_{22}, \quad \mathbb{Z}_{11}: \mathbb{Z}_{10}, \quad \mathrm{D}_{46}, \quad \mathbb{Z}_{23}: \mathbb{Z}_{22}
$$

Thus $|G|$ is square-free. By [13, Theorem 1.1], the triple $\left(G, \operatorname{Aut} \Gamma,(\operatorname{Aut} \Gamma)_{u}\right)$ lies in Table 1.

Case 2: Suppose that $G_{i} \leq \mathrm{D}_{\frac{2\left(r^{e} \pm 1\right)}{d}}$. Arguing as above, $N=T$. If $G_{i} \leq \mathrm{D}_{\frac{2\left(r^{e}-1\right)}{d}}$, then $q(q+1) / d$ divides 48 , which is a contradiction since $q>3$. Thus $G_{i} \leq \mathrm{D}_{\underline{2(r e+1)}}^{d}$. For this case, $q(q-1) / d$ divides 48 . Calculation shows $q=4$, and then $W=\mathbb{Z}_{5}$. By Case 1, this case is also excluded.

Case 3: Suppose that $G_{i} \leq \mathrm{A}_{5}$. Then $G_{i}=\mathrm{D}_{10}$. Arguing as Case 1, we obtain that $\ell=1$. Since $15 \mid q\left(q^{2}-1\right)$, it follows that $5|q, 5| q-1$ or $5 \mid q+1$.

Suppose that $5 \mid q$. Since $\frac{|X|}{\left|G_{1}\right|}$ divides $\left|X_{u}\right|$, we conclude that $q=5$. By Case 1 , this case does not occur. Similarly, we also exclude other cases.

Case 4: Suppose that $G_{i} \leq \operatorname{PGL}\left(2, r^{f}\right)$. Let $\hat{r}=\frac{r^{e-f}\left(r^{2 e}-1\right)}{r^{2 f}-1}$. Then $\hat{r}$ divides 48 . If $e-f=1$, then $e=2$ and $f=1$ as $f \mid e$. However, $\hat{r}$ does not divide 48, a contradiction. Thus $e>f+1$, and so $r=2$. Then $\frac{\left(r^{2 e}-1\right)}{r^{2 f}-1}=3$. Clearly, this is a contradiction.

In what follows, we will show $R=1$.

Lemma 4.6. The radical $R=1$.

Proof. Suppose that $R \neq 1$. Let $L=R \cap G$. Suppose that $L \neq 1$. Since $W$ is minimal in $G$, it follows that $W \leq L$. Since $X / R$ is insoluble, $\Gamma$ is a normal cover of $\Gamma_{R}$, see Lemma 2.4.

Let $\bar{G}=G R / R$. Then $\bar{G} \cong G /(G \cap R)$ is cyclic since $W \leq R$. Since $\Gamma$ is a Cayley graph of $G, \Gamma_{R}$ is a connected Cayley graph of $\bar{G}$. By [1, Theorem 1.2], we obtain either $\boldsymbol{\Gamma}_{\boldsymbol{R}} \cong \boldsymbol{K}_{\mathbf{4}}$ or $\boldsymbol{K}_{\mathbf{3}, \mathbf{3}}$. In either case, Aut $\Gamma_{R}$ is soluble. Since $X / R \leq \mathrm{Aut} \Gamma_{R}$, $X / R$ is soluble. So is $X$, which is a contradiction. Thus $L=1$.

Let $\bar{G}=G R / R$. Since $L=1$, we have $\bar{G} \cong G$. Let $\bar{X}=X / R$. Then $\bar{X}=\bar{G} \bar{X}_{u}$. Arguing as the proof of Lemma 4.5 with $\bar{X}=\bar{G} \bar{X}_{u}$ in place of $X=G X_{u}, \bar{X}$ is almost simple, and $\bar{G} \cong \mathrm{D}_{14}, \mathbb{Z}_{11}: \mathbb{Z}_{10}$ or $\mathbb{Z}_{23}: \mathbb{Z}_{22}$. By Table $1, X$ is almost simple, which is a contradiction. Thus $R=1$.

The assertion of Theorem 1.1 follows from Lemmas 4.1 and 4.44 .6 


\section{Acknowledgments}

This work was partially supported by the NNSF of China (11301468), the NSF of Yunnan Province (2013FB001), and Yunnan University's Research Innovation Fund for Graduate Students (ynuy201688).

\section{References}

[1] Y. G. Baik, Y. Q. Feng, H. S. Sim and M. Y. Xu, On the normality of Cayley graphs of abelian groups, Algebra Colloq. 5 (1998) 297-304.

[2] Y. Cheng and J. Oxley, On weakly symmetric graphs of order twice a prime, $J$. Combin. Theory Ser. B 42 (1987) 196-211.

[3] J. H. Conway, R. T. Curtis, S. P. Norton, R. A. Parker and R. A. Wilson, Atlas of Finite Groups (Cambridge Press, Oxford, 1985).

[4] A. S. Detinko and D. L. Flannery, Nilpotent primitive linear groups over finite fields, Comm. Algebra. 33 (2005) 497-505.

[5] S. F. Du and F. R. Wang, Arc-transitive cubic Cayley graphs on PSL $(2, p)$, Sci. China A 48 (2005) 1297-1308.

[6] Y. Q. Feng and J. H. Kwak, Cubic symmetric graphs of order a small number times a prime or a prime square, J. Combin. Theory Ser. B 97 (2007) 627-646.

[7] C. D. Godsil, On the full automorphism group of a graph, Combinatorica 10 (1981) 243-256.

[8] D. Gorenstein, Finite Groups (Harper and Row, 1968).

[9] B. Huppert, Endliche Gruppen I (Springer, Berlin, 1967).

[10] L. S. Kazarin, Groups that can be represented as a product of two solvable subgroups, Comm. Algebra 14 (1986) 1001-1066.

[11] C. H. Li, Isomorphisms of finite Cayley graphs, Ph.D. thesis, The University of Western Australia (1996).

[12] J. J. Li and Z. P. Lu, Cubic s-arc transitive Cayley graphs, Discrete Math. 209(20) (2009) 6014-6025.

[13] C. H. Li, Z. P. Lu and G. X. Wang, Vertex-transitive cubic graphs of square-free order, J. Graph Theory 75 (2014) 1-19.

[14] M. Suzuki, Group Theory I (Springer-Verlag, Berlin, 1982).

[15] M. Y. Xu, Automorphism groups and isomorphisms of Cayley digraphs, Discrete Math. 182 (1998) 309-319.

[16] S. J. Xu, X. G. Fang, J. Wang and M. Y. Xu, On cubic s-arc transitive Cayley graphs of finite simple groups, European J. Combin. 26 (2005) 133-143.

[17] S. J. Xu, X. G. Fang, J. Wang and M. Y. Xu, 5-Arc transitive cubic Cayley graphs on finite simple groups, European J. Combin. 28 (2007) 1023-1036.

[18] C. X. Zhou and Y. Q. Feng, Automorphism groups of connected cubic Cayley graphs of order 4p, Algebra Colloq. 14(2) (2007) 351-359. 